\title{
DIAGNÓSTICOS DE ENFERMAGEM E PROBLEMAS COLABORATIVOS MAIS COMUNS NA GESTAÇÃO DE RISCO
}

\author{
Helga Geremias Gouveia ${ }^{1}$ \\ Maria Helena Baena de Moraes Lopes ${ }^{2}$
}

Gouveia HG, Lopes MHBM. Diagnósticos de enfermagem e problemas colaborativos mais comuns na gestação de risco. Rev Latino-am Enfermagem 2004 março-abril; 12(2):175-82.

Este estudo identificou o perfil demográfico, os diagnósticos clínicos e obstétricos, os diagnósticos de enfermagem e problemas colaborativos mais comuns a gestantes de risco em um hospital da cidade de São Paulo, em São Paulo, Brasil. Os dados foram coletados utilizando-se um formulário baseado nos Padrões Funcionais de Saúde de Gordon. Os diagnósticos de enfermagem foram determinados de acordo com a taxonomia da North American Nursing Diagnosis Association (NANDA). Os diagnósticos de enfermagem encontrados em 50\% ou mais das gestantes foram: risco para infecção (90,1\%); manutenção da saúde alterada (84,5\%); conforto alterado (80,3\%); risco para amamentação ineficaz (59,2\%); padrões de sexualidade alterados (52,1\%); medo (52,1\%) e dor (50,7\%). Os problemas colaborativos encontrados em 50\% ou mais dos casos foram: CP: trabalho de parto prematuro (62\%); CP: taquicardia materna (54,9\%) e CP: hipotensão (54,9\%). Assim, com base nesses resultados será possível direcionar a assistência de enfermagem prestada a essas gestantes.

DESCRITORES: gravidez de alto risco; diagnóstico de enfermagem; saúde da mulher

\section{NURSING DIAGNOSES AND MOST COMMON COLLABORATION PROBLEMS IN HIGH-RISK PREGNANCY}

This study identified the demographic profile, obstetric and clinical diagnoses, nursing diagnosis and most common collaboration problem among pregnant women subject to high-risk at a hospital in São Paulo, Brazil. Data were collected by means of a form based on Gordon's Functional Health Patterns. Nursing diagnoses were determined on the basis of the NANDA (North American Nursing Diagnosis Association) taxonomy. The nursing diagnoses found in 50\% or more of the pregnant women were: risk for infection (90.1\%), altered health maintenance (84.5\%), altered comfort (80.3\%), risk of ineffective breastfeeding (59.2\%), altered sexuality patterns (52.1\%), fear (52.1\%) and pain (50.7\%). The collaboration problem found in $50 \%$ or more of the cases was: Potential Complication: Preterm labor (62.0\%), Potential Complication: Maternal tachycardia (54,9\%) and Potential Complication: Hypotension (54,9\%). Thus, these results will allow us to guide the nursing care rendered to these pregnant women.

DESCRIPTORS: pregnancy; high-risk; nursing diagnosis; women's health

\section{DIAGNÓSTICOS DE ENFERMERÍA Y PROBLEMAS COLABORATIVOS MÁS} COMUNES EN EL EMBARAZO DE ALTO RIESGO

Este estudio tiene como propuesta identificar el perfil demográfico, diagnósticos clínico y obstétrico, diagnósticos de enfermería y problemas colaborativos más comunes en el embarazo de alto riesgo del hospital de la ciudad de São Paulo, Brazil. Los datos fueron recolectados utilizándose un formulario basado en los padrones funcionales de salud de Gordon. Los diagnósticos de enfermería fueron determinados de acuerdo con la taxonomía da North American Nursing Diagnosis Association (NANDA). Los diagnósticos de enfermería encontrados en 50\% o más de las gestantes fueron: Riesgo para infección (90,1\%); Manutención de la salud alterada (84,5\%); Confort alterado (80,3\%); Riesgo para la lactancia ineficaz (59,2\%); Patrones de sexualidad alterados (52,1\%); Miedo (52,1\%) y Dolor (50,7\%). Los problemas colaborativos encontrados en $50 \%$ o más de los casos fueron: Trabajo de parto prematuro (62,0\%); Taquicardia materna (54,9\%) e Hipotensión (59,9\%). Así, con base en estos resultados será posible orientar la atención de enfermería prestada a estas gestantes.

DESCRIPTORES: embarazo de alto riesgo; diagnostico de enfermeria; salud de las mujeres

\footnotetext{
${ }^{1}$ Enfermeira, Especialista em Enfermagem Obstétrica, Mestre em Enfermagem - Pós-Graduação em Enfermagem do Departamento de Enfermagem da Faculdade de Ciências Médicas da Universidade Estadual de Campinas, e-mail: wake@uol.com.br; ${ }^{2}$ Enfermeira, Especialista em Enfermagem Obstétrica, Doutor em Ciências, Professor Associado da Faculdade de Ciências Médicas da Universidade Estadual de Campinas, e-mail: mhbaena@fcm.unicamp.br
} 
A ciência da enfermagem está baseada numa

Os serviços hospitalares, de forma geral, têm escassez de recursos humanos especializados, como é o caso das enfermeiras obstetras que representam menos de $1 \%$ dos cerca de 22 mil enfermeiros do Estado de São $\mathrm{Paulo}^{(1)}$. O atendimento à gravidez de risco, por outro lado, exige equipe médica e de enfermagem especializada devido à sua complexidade, não apenas considerando-se as patologias, mas, sobretudo, as repercussões sobre a dinâmica familiar, estado emocional; enfim, sobre a mulher, seu concepto e sua família, considerando-se os aspectos biopsicossocioculturais e espirituais.

A Real e Benemérita Sociedade Portuguesa de Beneficência - Hospital São Joaquim (RBSPB - Hospital São Joaquim) da cidade de São Paulo, SP, Brasil, é um hospital geral que possui, entre outros serviços, o atendimento à gestante de risco. Já foi implantada em alguns setores a sistematização da assistência de enfermagem e pretende-se implantá-la na unidade de internação obstétrica, utilizando o processo de enfermagem como método.

A ciência da enfermagem está baseada em ampla estrutura teórica e o processo de enfermagem é o método através do qual essa é aplicada à prática ${ }^{(2)}$. O seu propósito é de oferecer estrutura na qual as necessidades individuais do cliente, seja ele indivíduo, família ou comunidade, possam ser satisfeitas ${ }^{(2)}$. Utilizando os diagnósticos de enfermagem, de uma classificação como a da North American Nursing Diagnosis Association $(\text { NANDA })^{(3)}$, é possível, ainda, o uso de uma linguagem comum, de estrutura organizada.

Quanto à gestação de risco, sabe-se que, embora a gestação seja um fenômeno fisiológico, há pequena parcela de mulheres, denominada de gestantes de risco, que, por possuirem características específicas ou por sofrer algum agravo, apresenta maior probabilidade de evolução desfavorável, tanto para o feto como si mesma ${ }^{(4)}$. As gestantes de risco representam $15 \%$ do total de mulheres grávidas $^{(5)}$. Nas gestações de risco, as preocupações com o sucesso da gestação se acumulam frente às complicações às quais a mulher grávida está sujeita. Dessa forma, a sistematização da assistência de enfermagem é valiosa, porque pode levar à visão global da condição tanto da mulher quanto do concepto, favorecendo a continuidade da assistência e direcionando-a através de embasamento científico $^{(6)}$. ampla estrutura teórica, e o processo de enfermagem é um método, através do qual essa estrutura é aplicada à prática da enfermagem ${ }^{(2)}$. A participação do cliente nesse processo é de fundamental importância, pois oferece subsídios para o levantamento e validação dos dados, expressando seus reais problemas, procurando realizar troca de informações, de expectativas e de experiências. Assim, será possível desenvolver um plano de cuidados mais adequado e estabelecer resultados atingíveis.

O modelo bifocal da prática clínica identifica as respostas do cliente como diagnósticos de enfermagem ou problemas colaborativos que, juntos, compreendem o âmbito das respostas tratadas pela enfermagem e como tal definem a sua natureza exclusiva ${ }^{(7)}$.

Em 1990, na $9^{\text {th }}$ National Conference of the NANDA, foi aprovada a definição de diagnóstico de enfermagem como "um julgamento clínico sobre as respostas do indivíduo, família ou da comunidade a problemas de saúde/processos vitais reais ou potenciais. Um diagnóstico de enfermagem proporciona a base para a seleção de intervenções de enfermagem para atingir resultados pelos quais a enfermeira é responsável”(3). Os diagnósticos de enfermagem proporcionam, portanto, mecanismo útil para estruturar o conhecimento de enfermagem, na tentativa da definição do papel e domínio do enfermeiro ${ }^{(7)}$.

Por outro lado, problemas colaborativos são "certas complicações fisiológicas que as enfermeiras monitoram para detectar o estabelecimento ou a modificação subseqüente em seu estado. As enfermeiras controlam os problemas colaborativos usando intervenções prescritas pelo médico e/ou ela própria, para minimizar as complicações dos eventos" ${ }^{\text {(7) }}$. Os problemas colaborativos iniciam com o título diagnóstico 'complicação potencial' (CP), esse indica o foco da enfermagem para o problema colaborativo, que é reduzir a gravidade de certos fatores ou eventos fisiológicos ${ }^{(7)}$.

As gestantes de risco devem ser consideradas como um grupo que possui necessidades específicas, em que a esperança do sucesso da evolução da gestação até o termo se confronta com as complicações presentes ou potenciais. Há diagnósticos de enfermagem e problemas colaborativos que são comuns às gestantes de risco, independente do diagnóstico (clínico e/ou obstétrico) que estejam apresentando, o que permite 
estabelecer quais dados devem ser prioritariamente investigados no momento da admissão da gestante, quando, algumas vezes, o diagnóstico (clínico e/ou obstétrico) ainda não está definido. Por outro lado, o diagnóstico quando conhecido, é importante, pois há diagnósticos de enfermagem e problemas colaborativos que estão estreitamente relacionados a eles.

Com exceção de alguns livros-texto que descrevem os diagnósticos de enfermagem ${ }^{(8-10)}$ e problemas colaborativos na gestação de risco ${ }^{(7,11)}$, há poucos estudos na literatura nacional e internacional que abordam esse tema. Geralmente é enfocada uma situação específica como a hipertensão gestacional ${ }^{(12-14)}$. Assim, faz-se necessário o desenvolvimento de estudos que enfoquem, na gestação de risco, os diagnósticos de enfermagem e problemas colaborativos.

\section{OBJETIVOS}

Ao desenvolver-se este trabalho pretendeu-se identificar os diagnósticos de enfermagem e os problemas colaborativos de gestantes de risco no Real e Benemérita Sociedade Portuguesa de Beneficência - Hospital São Joaquim, São Paulo, SP, Brasil, além de:

- identificar o perfil demográfico, os diagnósticos clínicos e obstétricos de gestantes de risco atendidas naquele hospital;

- identificar a freqüência dos diagnósticos de enfermagem das gestantes de risco e de acordo com os diagnósticos médicos (clínicos e/ou obstétricos) e,

- identificar a freqüência dos problemas colaborativos das gestantes de risco e de acordo com os diagnósticos médicos (clínicos e/ou obstétricos).

\section{SUJEITOS E MÉTODOS}

A população foi composta por gestantes de risco internadas por motivo clínico e/ou obstétrico no período de abril a setembro de 2000. Trata-se de estudo descritivo, exploratório e transversal e, para o cálculo do tamanho da amostra, estabeleceu-se amostragem sistematizada, na qual a cada duas internações uma seria considerada para o levantamento de dados.

Foi elaborado formulário com questões abertas e fechadas, baseado nos Padrões Funcionais de Saúde ${ }^{(15)}$, contendo questões relacionadas a cada padrão funcional e dados obstétricos pertinentes, que foi avaliado por uma enfermeira obstetra especialista em diagnóstico de enfermagem e, após, foi aplicado a 10 clientes. Como não houve necessidade de alterações, deu-se continuidade à coleta de dados. Os dados foram obtidos através de uma única entrevista, realizada dentro das primeiras 24 horas de internação, por uma só entrevistadora. Foi estabelecido esse período de tempo, considerando-se a necessidade de estabelecer um plano inicial de atendimento às gestantes no momento em que são internadas, visando atender as necessidades mais imediatas. Além disso, optou-se por realizar a coleta de dados num único momento a fim de viabilizar o estudo, uma vez que o tempo de entrevista durava cerca de uma hora.

Para análise dos dados, foram calculadas as freqüências absolutas e relativas das variáveis de caracterização da amostra (idade, escolaridade, situação conjugal, ocupação, número de gestações, paridade e número de abortos). Os diagnósticos de enfermagem e os problemas colaborativos foram determinados a partir dos dados contidos no formulário, por três especialistas em Enfermagem Obstétrica, duas delas com mais de dez anos de experiência no uso dos diagnósticos de enfermagem da NANDA, sendo a terceira a entrevistadora. Uma vez identificados os dados relevantes, com base apenas nos dados registrados, esses foram comparados com as características definidoras e problemas colaborativos relatados na literatura ${ }^{(3,11)}$. Os diagnósticos de enfermagem e problemas colaborativos somente foram considerados presentes, quando havia consenso entre as especialistas. Os diagnósticos de enfermagem e problemas colaborativos foram categorizados de acordo com sua freqüência e apresentados considerando-se primeiramente a amostra como um todo e, posteriormente, de acordo com o diagnóstico clínico e/ou obstétrico.

Foram considerados no presente estudo os diagnósticos de enfermagem e características definidoras aprovados na $12^{\mathrm{a}}$ Reunião da NANDA ${ }^{(3)}$ e acrescentou-se a esses, o diagnóstico risco para amamentação ineficaz $^{(7,11)}$, que deve ser investigado desde o período prénatal, a fim de serem tomadas medidas preventivas, e os diagnósticos conforto alterado e risco para transmissão de infecção $0^{(11)}$. Quanto aos problemas colaborativos, foram considerados aqueles propostos na literatura ${ }^{(11)}$ e 
acrescentados: CP: taquicardia materna e CP: hipotensão, por serem relativamente freqüentes em pacientes que usam drogas tocolíticas.

Todas as participantes do estudo assinaram um termo de consentimento livre e esclarecido e a pesquisa foi aprovada pelo Comitê de Ética em Pesquisa da instituição.

\section{RESULTADOS}

No período de abril a setembro de 2000 , foram entrevistadas 71 gestantes de risco que representaram $50 \%$ do total das gestantes de risco internadas no período.

Em relação aos dados de caracterização da amostra, cerca de $50 \%$ das gestantes eram jovens, 12 $(16,9 \%)$ adolescentes, na faixa dos 15 aos 19 anos e 24 $(33,8 \%)$ adultas jovens, entre 20 e 24 anos. A menor idade foi 15 anos e a maior, 40 anos; a idade média foi de 25,6 anos ( $\pm 6,6$ anos), com mediana de 24 anos.

Quanto à escolaridade, $2(2,8 \%)$ tinham curso superior completo e 5 (7\%), incompleto; $18(25,4 \%)$ tinham ensino médio completo e 11 (15,5\%), incompleto; 7 (9,9\%) tinham o ensino fundamental completo e 26 (36,6\%), incompleto e $2(2,8 \%)$ eram analfabetas.

Em relação à situação conjugal, das 12 gestantes que eram adolescentes, $8(66,7 \%)$ moravam com seus parceiros e $4(33,3 \%)$ não. Dentre as demais gestantes, $50(84,7 \%)$ moravam com seu parceiro, 7 (11,9\%) não moravam e $2(3,4 \%)$ não tinham parceiro.

No que se refere à atividade profissional, estudo ou ocupação, 35 (49,3\%) não exerciam trabalho fora do lar e aquelas que tinham trabalho remunerado exerciam profissões ou ocupações diversas tais como: vendedora, empregada doméstica, auxiliar administrativo, auxiliar de enfermagem, entre outros. Sete $(9,8 \%)$ gestantes eram estudantes e dessas, seis, além do estudo, realizavam atividades domésticas e nenhuma trabalhava fora do lar.

$\mathrm{Na}$ história obstétrica pregressa, em relação ao número de gestações, 25 (35,2\%) estavam grávidas pela primeira vez e $46(64,8 \%)$ eram multigestas. Dentre as gestantes adolescentes, 9 (75\%) eram primigestas e 3 (25\%) eram multigestas, sendo que duas se encontravam em sua segunda gestação e uma, com 19 anos, na quarta gestação. Quanto aos partos e/ou abortos anteriores, 28 gestantes $(60,9 \%)$ tinham partos anteriores e nenhum aborto, $12(26,1 \%)$ tinham parto e pelo menos um aborto e $6(13 \%)$ tinham tido somente abortos. O grupo como um todo teve 75 partos anteriores e, desses, 45 partos $(60 \%)$ tinham sido por via vaginal (42 normais e três fórceps) e 30 (40\%), cesarianas. Em relação às gestantes que tiveram abortos (18 casos), 14 (78\%) abortaram espontaneamente, uma provocou o aborto e $3(16,5 \%)$ tiveram tanto abortos provocados quanto espontâneos.

Quanto aos diagnósticos médicos (clínicos e/ou obstétricos) encontrados no grupo estudado, observamos, através da Tabela 1, que o trabalho de parto prematuro foi aquele que teve maior incidência com $29(40,8 \%)$ casos, sendo que em nove gestações estava associado a outros diagnósticos médicos. Nove gestantes (12,7\%) apresentavam doença hipertensiva específica da gestação (DHEG) e 6 (8,5\%), o diagnóstico de hipertensão que, em apenas um caso, foi especificado como hipertensão arterial sistêmica. Optou-se por agrupar todos os 15 casos em uma só categoria denominada 'DHEG/hipertensão', uma vez que não foi possível especificar o tipo de hipertensão. A amniorrexe prematura esteve presente em 9 (12,7\%) casos, em uma das vezes associada ao trabalho de parto prematuro e, em outra, a oligoâmnio. A infecção do trato urinário foi constatada em 4 (5,6\%) casos e houve $3(4,2 \%)$ casos de pielonefrite, que foram agrupados em um mesmo grupo para fins de análise.

Tabela 1 - Diagnósticos clínicos e obstétricos das gestantes de risco da unidade de internação obstétrica do RBSPB - Hospital São Joaquim. São Paulo, SP, 2000

\begin{tabular}{lccc}
\hline Diagnósticos clínicos e obstétricos & $\mathbf{n}$ & $\%^{\star}$ & $\%^{\star \star}$ \\
\hline Trabalho de parto prematuro & 29 & 34,4 & 40,8 \\
DHEG/hipertensão & 15 & 17,8 & 21,1 \\
Aminiorrexe prematura & 9 & 10,7 & 12,7 \\
Infecção do trato urinário & 4 & 4,7 & 5,6 \\
Pielonefrite & 3 & 3,6 & 4,2 \\
Ameaça de aborto & 3 & 3,6 & 4,2 \\
Malformação fetal & 3 & 3,6 & 4,2 \\
Hiperêmese gravídica & 2 & 2,4 & 2,8 \\
Incompetência istmo-cervical & 2 & 2,4 & 2,8 \\
Diagnóstico a esclarecer & 2 & 2,4 & 2,8 \\
Diabetes & 2 & 2,4 & 2,8 \\
Oligoâmnio & 2 & 2,4 & 2,8 \\
Coriorrexe & 1 & 1,2 & 1,4 \\
Retardo de crescimento intra-útero & 1 & 1,2 & 1,4 \\
Gemelaridade & 1 & 1,2 & 1,4 \\
Placenta prévia & 1 & 1,2 & 1,4 \\
Infecção pelo papiloma vírus humano & 1 & 1,2 & 1,4 \\
Cardiopatia fetal & 1 & 1,2 & 1,4 \\
Neoplasia tubária & 1 & 1,2 & 1,4 \\
Bartolinite & 1 & 1,2 & 1,4 \\
Total & $\mathbf{8 4}$ & $\mathbf{1 0 0}$ & $\mathbf{1 0 0}$ \\
\hline
\end{tabular}

Legenda: * \% do diagnóstico; ** \% de gestantes com o diagnóstico

Os diagnósticos de enfermagem foram agrupados de acordo com sua freqüência e são apresentados na Tabela 2. 
Tabela 2 - Distribuição dos diagnósticos de enfermagem, identificados entre gestantes de risco da unidade de internação obstétrica do RBSPB - Hospital São Joaquim. São Paulo, 2000

\begin{tabular}{|c|c|c|}
\hline Diagnóstico de Enfermagem & $\mathbf{n}$ & $\%$ \\
\hline 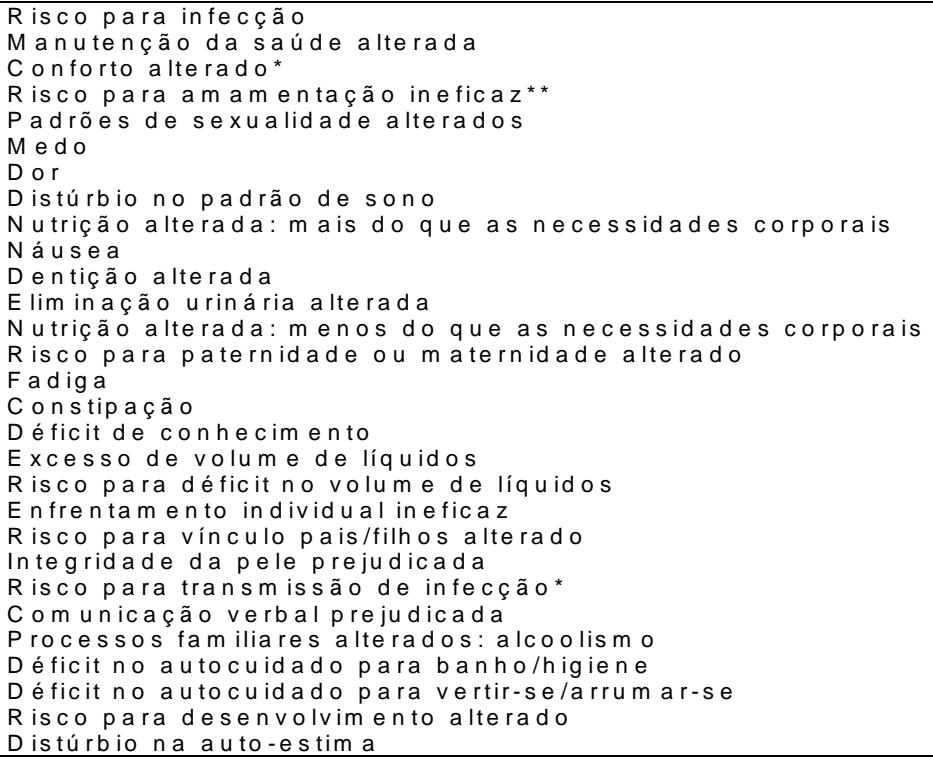 & $\begin{array}{ll}6 & 4 \\
6 & 0 \\
5 & 7 \\
4 & 2 \\
3 & 7 \\
3 & 7 \\
3 & 6 \\
2 & 9 \\
2 & 4 \\
2 & 1 \\
2 & 0 \\
2 & 0 \\
1 & 3 \\
1 & 2 \\
1 & 2 \\
9 & \\
7 \\
3 \\
3 \\
3 \\
2 \\
2 \\
1 \\
1 \\
1 \\
1 \\
1 \\
1 \\
1 \\
\end{array}$ & $\begin{array}{r}90,1 \\
84,5 \\
80,3 \\
59,2 \\
52,1 \\
52,1 \\
50,7 \\
40,8 \\
33,8 \\
29,6 \\
28,2 \\
28,2 \\
18,3 \\
16,9 \\
16,9 \\
12,7 \\
9,9 \\
4,2 \\
4,2 \\
4,2 \\
2,8 \\
2,8 \\
1,4 \\
1,4 \\
1,4 \\
1,4 \\
1,4 \\
1,4 \\
1,4 \\
\end{array}$ \\
\hline
\end{tabular}

* Diagnóstico de enfermagem que atualmente não está na lista da NANDA, mas é referenciado por alguns autores ${ }^{(7,11)}$

** Diagnóstico de enfermagem que atualmente não está na lista da NANDA, mas é referenciado por alguns autores ${ }^{(7-8,11)}$

Na Tabela 3, procurou-se considerar a freqüência dos diagnósticos de enfermagem de acordo com os diagnósticos (clínicos e/ou obstétricos) mais freqüentes, a saber, trabalho de parto prematuro, DHEG/hipertensão, aminiorrexe prematura e infecção do trato urinário/ pielonefrite. A freqüência dos problemas colaborativos de um modo geral, e de acordo com os diagnósticos (clínicos e/ou obstétricos) mais freqüentes, são apresentados na Tabela 4.

Tabela 3 - Distribuição das gestantes de risco da unidade de internação obstétrica do RBSPB - Hospital São Joaquim, segundo os diagnósticos de enfermagem dos casos de um modo geral e nos casos de trabalho de parto prematuro, DHEG/hipertensão, aminiorrexe prematura e infecção do trato urinário/pielonefrite. São Paulo, 2000

\begin{tabular}{|c|c|c|c|c|c|c|c|c|c|c|}
\hline \multirow[t]{2}{*}{ Diagnósticos de enfermagem } & \multicolumn{2}{|c|}{ Geral } & \multicolumn{2}{|c|}{ A } & \multicolumn{2}{|c|}{ B } & \multicolumn{2}{|c|}{ C } & \multicolumn{2}{|c|}{ D } \\
\hline & $\mathbf{n}$ & $\%$ & $\mathbf{n}$ & $\%$ & $\mathbf{n}$ & $\%$ & $\mathbf{n}$ & $\%$ & $\mathbf{n}$ & $\%$ \\
\hline $\mathrm{R}$ isco para infecção & 64 & 90,1 & 27 & 93,1 & 13 & 86,6 & 9 & 100,0 & 5 & 71,4 \\
\hline Manutenção da saúde a lterada & 60 & 84,5 & 24 & 82,8 & 13 & 86,6 & 8 & 88,9 & 7 & 100,0 \\
\hline Conforto alterado* & 57 & 80,3 & 23 & 79,3 & 12 & 80,0 & 7 & 77,8 & 6 & 85,7 \\
\hline Risco para am am entação inefica $z^{* *}$ & 42 & 59,2 & 21 & 72,4 & 6 & 40,0 & 7 & 77,8 & 3 & 42,8 \\
\hline Padrões de sexualidade alterados & 37 & 52,1 & 19 & 65,5 & 6 & 40,0 & 3 & 33,3 & 3 & 42,8 \\
\hline Medo & 37 & 52,1 & 17 & 58,6 & 7 & 46,6 & 8 & 88,9 & - & - \\
\hline Dor & 36 & 50,7 & 20 & 69,0 & 4 & 26,6 & 5 & 55,6 & 3 & 42,8 \\
\hline D istúrbio no padrão do sono & 29 & 40,8 & 13 & 44,8 & 6 & 40,0 & 6 & 66,7 & 1 & 14,2 \\
\hline $\begin{array}{l}\text { Nutrição alterada: mais do que as } \\
\text { necessidades corporais }\end{array}$ & 24 & 33,8 & 8 & 27,6 & 12 & 80,0 & 2 & 22,2 & - & - \\
\hline $\mathrm{N}$ á us e a & 21 & 29,6 & 6 & 20,7 & 4 & 26,6 & 2 & 22,2 & 1 & 14,2 \\
\hline Elim in ação urinária a lte ra da & 20 & 28,2 & 9 & 31,0 & 3 & 20,0 & 2 & 22,2 & 5 & 71,4 \\
\hline Dentição a lterad a & 20 & 28,2 & 8 & 27,6 & 5 & 33,3 & - & - & 2 & 28,5 \\
\hline $\begin{array}{l}\text { Nutrição alterada: menos do que as } \\
\text { necessidades corporais }\end{array}$ & 13 & 18,3 & 7 & 24,1 & - & - & 2 & 22,2 & 3 & 42,8 \\
\hline $\begin{array}{l}\text { Risco para paternidade/m aternidade } \\
\text { alterada }\end{array}$ & 12 & 16,9 & 6 & 20,7 & 2 & 13,3 & 2 & 22,2 & 1 & 14,2 \\
\hline F a dig a & 12 & 16,9 & 4 & 13,8 & 2 & 13,3 & 1 & 11,1 & 1 & 14,2 \\
\hline Constip ação & 9 & 12,6 & 2 & 6,9 & 1 & 6,6 & 3 & 33,3 & 1 & 14,2 \\
\hline Déficit de conhecimento & 7 & 9,9 & 3 & 10,3 & - & - & 3 & 33,3 & 2 & 28,5 \\
\hline Excesso de volum e de líquidos & 3 & 4,2 & - & - & 3 & 20,0 & - & - & - & - \\
\hline Risco para déficit do volume de líquidos & 3 & 4,2 & & - & - & - & - & - & - & - \\
\hline Enfrentam ento individual in eficaz & 3 & 4,2 & 1 & 3,4 & - & - & 1 & 11,1 & - & - \\
\hline $\mathrm{R}$ isco para vínculo pais/filhos alterado & 2 & 2,8 & - & - & - & - & - & - & & - \\
\hline Integridade da pele prejudicada & 2 & 2,8 & - & - & 1 & 6,6 & - & - & 1 & 14,2 \\
\hline Déficit no autocuidado parabanho/higiene & 1 & 1,4 & 1 & 3,4 & - & - & - & - & - & - \\
\hline Défic it no autocuidado para vestir- & 1 & 1,4 & 1 & 3,4 & - & - & - & - & - & - \\
\hline se/arrum ar-se & & & & & & & & & & \\
\hline Processos fam iliares alterados: a lcoolis mo & 1 & 1,4 & - & - & - & - & - & - & - & - \\
\hline$R$ isco para desenvolvim ento alterado & 1 & 1,4 & - & - & - & - & - & - & - & - \\
\hline D istúrbio na auto-estim a & 1 & 1,4 & - & - & - & - & - & - & - & - \\
\hline Comunicação verbal prejudicada & 1 & 1,4 & - & - & - & - & - & - & - & - \\
\hline$R$ isco paratransm issão de infeção & 1 & 1,4 & - & - & - & - & - & - & 1 & 14,2 \\
\hline
\end{tabular}

* Diagnóstico de enfermagem que atualmente não está na lista da NANDA, mas é referenciado por alguns autores ${ }^{(7,11)}$

** Diagnóstico de enfermagem que atualmente não está na lista da NANDA, mas é referenciado por alguns autores ${ }^{(7-8,11)}$

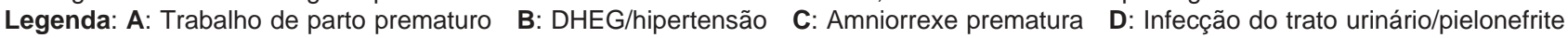


Tabela 4 - Distribuição das gestantes de risco da unidade de internação obstétrica do RBSPB - Hospital São Joaquim, segundo os problemas colaborativos dos casos de um modo geral e nos casos de trabalho de parto prematuro, DHEG/ hipertensão, amniorrexe prematura e infecção do trato urinário/pielonefrite. São Paulo, 2000

\begin{tabular}{|c|c|c|c|c|c|c|c|c|c|c|}
\hline \multirow[t]{2}{*}{ Problema colaborativo } & \multicolumn{2}{|c|}{ Geral } & \multicolumn{2}{|r|}{ A } & \multicolumn{2}{|c|}{ B } & \multicolumn{2}{|c|}{ C } & \multicolumn{2}{|c|}{ D } \\
\hline & $\mathbf{n}$ & $\%$ & $\mathbf{n}$ & $\%$ & $\mathbf{n}$ & $\%$ & $\mathbf{N}$ & $\%$ & $\mathbf{n}$ & $\%$ \\
\hline CP: Trabalho de parto prematuro & 44 & 62,0 & 29 & 100,0 & 1 & 6,6 & 9 & 100,0 & 7 & 100,0 \\
\hline CP: Taquicardia materna* & 39 & 54,9 & 29 & 100,0 & 1 & 6,6 & 9 & 100,0 & 2 & 28,6 \\
\hline CP: Hipotensão* & 39 & 54,9 & 29 & 10,0 & 1 & 6,6 & 9 & 100,0 & 2 & 28,6 \\
\hline CP: Sofrimento fetal & 27 & 38,0 & 3 & 10,3 & 15 & 100,0 & 9 & 100,0 & - & - \\
\hline CP: Insuficiência renal & 18 & 25,4 & 1 & 3,4 & 15 & 100,0 & - & - & 3 & 42,9 \\
\hline CP: Hipertensão & 16 & 22,5 & 2 & 6,9 & 15 & 100,0 & - & - & - & - \\
\hline CP: Convulsão & 15 & 21,1 & 1 & 3,4 & 15 & 100,0 & - & - & - & - \\
\hline CP: Proteinúria & 15 & 21,1 & 1 & 3,4 & 15 & 100,0 & - & - & - & - \\
\hline CP: Perturbação visual & 15 & 21,1 & 1 & 3,4 & 15 & 100,0 & - & - & - & - \\
\hline CP: Desidratação & 3 & 4,2 & - & - & - & - & - & - & - & - \\
\hline CP: Abortamento & 3 & 4,2 & - & - & - & - & - & - & - & - \\
\hline CP: Ruptura das membranas amnióticas & 3 & 4,2 & 1 & 3,4 & - & - & - & - & - & - \\
\hline CP: Hiperglicemia/hipoglicemia & 2 & 2,8 & 1 & 3,4 & 1 & 6,6 & - & - & - & - \\
\hline CP: Sangramento pré-natal & 1 & 1,4 & - & - & - & - & - & - & - & - \\
\hline CP: Hemorragia pós-parto & 1 & 1,4 & - & - & - & - & - & - & - & - \\
\hline
\end{tabular}

Legenda: A: Trabalho de parto prematuro $\quad$ B: DHEG/hipertensão $\quad$ C: Amniorrexe prematura $\quad$ D: Infecção do trato urinário/pielonefrite ${ }^{*}$ Acrescentados aos problemas colaborativos ${ }^{(7,11)}$

\section{DISCUSSÃO}

Considerando-se os diagnósticos de enfermagem mais freqüentes na gestação, segundo alguns autores ${ }^{(8-}$ $9,11,16)$, verifica-se que no presente estudo todos os diagnósticos que tiveram freqüência maior que $50 \%$, apesar de terem sido identificados em gestantes de risco, também são encontrados na gestação de baixo risco, com exceção do diagnóstico 'risco para amamentação ineficaz', visto que esse não faz parte da classificação diagnóstica da NANDA, embora tenha sido sugerido ou estudado por alguns autores ${ }^{(7-8)}$.

Comparando-se os dados com um estudo semelhante sobre diagnósticos de enfermagem em gestações de risco, desenvolvido em Campinas (SP) ${ }^{(17)}$, pudemos verificar que os resultados são similares, diferindo apenas quanto aos diagnósticos de hipertemia, mobilidade física prejudicada, padrão respiratório ineficaz, diarréia, reação de pesar antecipada e senso-percepção alterada que não foram identificados no presente estudo. Em parte, essas diferenças podem ser explicadas pelo fato de que, no estudo citado, foram utilizadas, como referência, a adaptação ao nosso meio da Taxonomia I da NANDA $^{(18)}$, na qual foram realizadas algumas modificações, acréscimos e translocações. Assim, por exemplo, a restrição ao leito imposta por prescrição médica, comum nos casos de trabalho de parto prematuro, foi considerada como evidência para mobilidade física prejudicada e, no presente estudo, situações semelhantes foram consideradas como déficit no autocuidado para banho/higiene e déficit no autocuidado para vestir-se/ arrumar-se.

Alguns estudos sobre diagnósticos de enfermagem em gestantes de risco foram desenvolvidos considerando um determinado diagnóstico médico como, por exemplo, trabalho de parto prematuro, hipertensão gestacional, entre outros. Em vista disso, serão discutidos, a seguir, os diagnósticos de enfermagem de acordo com os diagnósticos médicos clínicos e/ou obstétricos mais freqüentes.

Os diagnósticos de enfermagem dos casos de trabalho de parto prematuro que estiveram presentes em $50 \%$ ou mais dos casos como, por exemplo, conforto alterado e medo e aqueles com menor freqüência como constipação, distúrbio no padrão de sono, risco para maternidade/paternidade alterados e déficit de conhecimento, também foram referidos por outros autores $^{(9)}$. O distúrbio no padrão de sono, no presente estudo, esteve associado à dor em 13 casos. Esse é um dado esperado, visto que existe associação entre esses dois diagnósticos ${ }^{(19)}$.

Quanto aos casos de DHEG/hipertensão, os diagnósticos de enfermagem do presente estudo que tiveram mais de $50 \%$ de freqüência também foram citados por outros autores ${ }^{(9-14)}$, exceto conforto alterado. Outros diagnósticos referidos pela literatura como medo, distúrbio no padrão de sono e excesso de volume de líquido também foram relativamente freqüentes, sendo que os dois primeiros podem estar relacionados à possibilidade de perda fetal e o último, à fisiopatologia da doença hipertensiva 
da gestação, que é caracterizada por hipertensão, edema e proteinúria ${ }^{(20)}$. O diagnóstico dentição alterada não foi citado por nenhum dos autores, por ter sido aprovado pela NANDA apenas em 1998. Quando comparamos os dados do presente estudo com outros realizados no Brasil, verificamos que somente três diagnósticos citados por outros autores $^{(13-14)}$ não foram encontrados na população aqui estudada, a saber, ansiedade, mobilidade física prejudicada e déficit de conhecimento. Alguns autores ${ }^{(10)}$ utilizaram terminologia diferente, como alteração no volume de líquido, ou não especificaram se nutrição alterada era mais ou menos do que as necessidades corporais, o que dificulta a comparação entre os dados. Alteração na perfusão tissular de nível placentário não foi considerado no presente estudo, visto que o foco desse tratamento é eminentemente médico. Dessa forma, optou-se por considerar as evidências como relacionadas ao problema colaborativo 'CP: sofrimento fetal' e não a um diagnóstico de enfermagem. Alguns autores citam a ansiedade e medo como diagnósticos comuns nesse grupo de gestantes. Em nosso estudo, consideramos que quando as gestantes expressavam percepção de ameaça (real ou imaginária), seria considerado como medo e não ansiedade, conforme propõem alguns autores ${ }^{(21)}$, como forma de distinguir esses dois diagnósticos.

Quanto aos diagnósticos de enfermagem de gestantes com amniorrexe prematura, constatamos que somente o diagnóstico risco para infecção teve concordância com outros autores ${ }^{(9)}$, visto que o diagnóstico risco para infecção é absolutamente claro e bem determinado nos casos de ruptura das membranas amnióticas.

Em relação aos diagnósticos de enfermagem das gestantes com infecção do trato urinário e pielonefrite, não foram encontrados na literatura estudos envolvendo gestantes com esses diagnósticos médicos. Dois diagnósticos encontrados em nosso estudo também são observados em pessoas não grávidas ${ }^{(11)}$ : risco para nutrição alterada: menos do que as necessidades corporais e conforto alterado; esse último foi identificado por nós com freqüência superior a $50 \%$.

Nos problemas colaborativos incluiu-se taquicardia materna e hipotensão que não constam na classificação proposta por outro autor ${ }^{(7,11)}$ visto que, nos casos de trabalho de parto prematuro e amniorrexe prematura, geralmente são administradas drogas tocolíticas que podem causar essas alterações, como o cloridrato de isoxsuprina.
Vale salientar que entre as gestantes com trabalho de parto prematuro e DHEG/hipertensão, alguns problemas colaborativos estão relacionados a outros quadros clínicos e/ou obstétricos, considerando que, em alguns casos, as gestantes apresentavam mais de um diagnóstico médico.

\section{CONCLUSÕES E CONSIDERAÇÕES FINAIS}

A amostra estudada foi caracterizada por mulheres jovens, multigestas, com média escolaridade, com parceiros com os quais coabitavam, sendo que apenas metade delas trabalhava fora do lar. Os diagnósticos médicos (clínico ou obstétrico) de maior freqüência foram: trabalho de parto prematuro, doença hipertensiva específica da gestação/hipertensão, aminiorrexe prematura e infecção do trato urinário/pielonefrite.

Os diagnósticos de enfermagem encontrados em $50 \%$ ou mais das mulheres, e que devem ser priorizados independentemente do diagnóstico médico, foram: risco para infecção, manutenção da saúde alterada, conforto alterado, dor, risco para amamentação ineficaz, padrões de sexualidade alterados e medo. Cabe ressaltar que os quatro primeiros diagnósticos tiveram elevada freqüência entre as gestantes portadoras dos problemas médicos mais freqüentes.

Os problemas colaborativos encontrados em $50 \%$ ou mais dos casos foram: CP: trabalho de parto prematuro; CP: taquicardia materna e CP: hipotensão (54,9\%).

Por outro lado, alguns diagnósticos de enfermagem e problemas colaborativos apresentaram baixa freqüência, mas podem ser também significativamente importantes para a assistência de enfermagem à gestante de risco. Os dados evidenciam ainda que a gestante de risco além de suas necessidades específicas, decorrentes de sua situação clínica e/ou obstétrica, é uma mulher com necessidades próprias do estado gestacional.

Espera-se que os dados desta pesquisa ajudem os profissionais de enfermagem a praticar assistência à gestante de risco com melhor qualidade e com resultados mais eficazes. Para o hospital, onde foi desenvolvido este trabalho, os diagnósticos de enfermagem e problemas colaborativos identificados vão nortear a assistência de enfermagem à gestante de risco, visando a coleta de dados e a prestação de cuidados mais direcionados aos problemas comuns a esse grupo. Contudo, é iminente 
atentar às necessidades individuais de cada mulher, de forma a personalizar o atendimento. Sabe-se que esse é um desafio que requer o envolvimento não só da diretoria de enfermagem como do grupo de enfermeiros e equipe

\section{REFERÊNCIAS BIBLIOGRÁFICAS}

1. Dias MA, Cunha AMCA. Sistematização da assistência de enfermagem: da teoria à prática. São Paulo (SP): CORENSP; 1999.

2. Iyer PW, Taptich BJ, Bernocchi-Losey D. Processo e diagnóstico em enfermagem. Porto Alegre (RS): Artes Médicas; 1993.

3. North American Nursing Association. Diagnósticos de enfermagem da NANDA: definições e classificação 19992000. Porto Alegre (RS): Artes Médicas; 2000.

4. Ministério da Saúde (BR). Gestação de alto risco. Brasília (DF): Ministério da Saúde [online] 2000 [acessado em 19 Ago. 2000]; Disponível em: URL:http://www.saude.gov.br/ Programas/mulher/Gestao.htm

5. Ministério da Saúde (BR). Emergências. Brasília (DF): Ministério da Saúde [online] 2001 [acessado em 26 Jul. 2001]; Disponível em: URL:http//www.saude.gov.br/Programas/ mulher/Emergencias.htm

6. Waldow VR. Processo de enfermagem: teoria e prática. Rev Gaúcha Enferm 1988 janeiro; 9(1):14-22.

7. Carpenito LJ. Diagnóstico de enfermagem - aplicação à prática clínica. 6ํㅡㄹ ed. Porto Alegre (RS): Artes Médicas; 1997. 8. Vale IN. Risco para amamentação ineficaz: proposta de diagnóstico de enfermagem. [tese]. São Paulo (SP): Escola de Enfermagem/USP; 1999.

9. Hanson-Smith B. Nursing care planning guides for childbearing families. $4^{\text {th }}$ ed. Baltimore (Maryland): Williams \& Wilkins; 1994

10. Dickason EJ, Schult MO. Clinical companion to maternalinfant nursing care. St. Louis (Missouri): Mosby; 1998.

11. Carpenito LJ. Manual de diagnósticos de enfermagem. $6^{\underline{a}}$ ed. Porto Alegre (RS): Artes Médicas; 1999.

12. Silva AS. Doença hipertensiva específica da gestação: proposta de assistência de enfermagem à gestante. [dissertação]. João Pessoa (PB): Centro de Ciências da Saúde/UFPB; 1996.

13. Aragão KS, Santos ZMSA. Sistematização da assistência de enfermagem à gestante com DHEG. Anais do 52 CBEn; 2000, outubro 21-26; Recife (PE): ABEn; 2000. p.323.

14. Albuquerque ALBL, Gonçalves GAA, Cruz MJN, Oliveira MR. Identificando diagnósticos de enfermagem em uma gestante acometida de doença hipertensiva específica da gestação (DHEG). Anais do 52 CBEn; 2000, outubro 21-26; Recife (PE): ABEn; 2000. p.538.

15. Gordon M. Nursing diagnosis: process and application. $3^{\text {rd }}$ ed. St. Louis (Missouri): Mosby; 1994.

16. Mazzo MHSN. Identificação de diagnósticos de enfermagem em gestantes com base na teoria das necessidades humanas básicas. [dissertação]. João Pessoa (PB): Centro de Ciências da Saúde/UFPB; 1997.

17. Carvalho LH, Nomellini PF, Fâncio, SA, Lopes MHBM. Diagnósticos de enfermagem em uma unidade de internação de gestantes de alto risco, segundo a taxonomia da NANDA. Anais do 48ํㅡ룽 1996, outubro 6-11; São Paulo (SP): ABEN; 1996. p.416. de enfermagem para que o processo se inicie e se mantenha, como tem sido demonstrado nas experiências de implantação do processo de enfermagem em outras instituições ${ }^{(22)}$.

18. Nobrega MML, Garcia TR. Uniformização da linguagem dos diagnósticos de enfermagem da NANDA. Sistematização das propostas do II SNDE. João Pessoa (PB): A União; 1994. 19. Cruz DALM, Pimenta CAM. Câncer e dor: alterações nos padrões de resposta humana. Acta Paul Enfermagem 1994 janeiro/março;7(1):27-4.

20. Neme B. Obstetrícia básica. São Paulo (SP): Sarvier; 1995. 21. Levin RF, Miller BK. Refinement of anxiety/fear nursing diagnoses. In: Rantz MI, Lemone P, ed. Classification of Nursing Diagnosis. $13^{\text {th }}$ National Conference of the North American Nursing Diagnosis Association; 1999, April 22-26; St. Louis, Missouri. St. Louis: Cinahl Information Systems; 1999. p. 484.

22. Lopes MHBM. Experiência de implantação do processo de enfermagem utilizando os diagnósticos de enfermagem (Taxonomia NANDA), resultados esperados, intervenções e problemas colaborativos. Rev Latino-am Enfermagem 2000 julho; 8(3):115-8. 